\title{
SISAR Imaging - Radio Holography Signal Reconstruction Based on Receiver-Transmitter Motion
}

\author{
Ilias Theodorou ${ }^{1}$, Christos V. Ilioudis ${ }^{1}$, Carmine Clemente $^{1}$, Massimiliano Vasile ${ }^{2}$ \\ University of Strathclyde, Glasgow, UK \\ Email: $\{$ ilias.theodorou, c.ilioudis, carmine.clemente, massimiliano.vasile $\} @$ strath.ac.uk
}

\begin{abstract}
Radio Holography Signal (RHS) reconstruction is the primary method used for Shadow Inverse Synthetic Aperture Radar (SISAR) imaging algorithm application. In this paper an alternative way for RHS signal reconstruction is introduced based on the motion of receiver and/or transmitter ends. In particular, a novel model for time domain RHS signal is derived assuming a moving-ends (ME) scenario followed by the RHS reconstruction method. The model and reconstruction method area assessed through simulated data.
\end{abstract}

\section{INTRODUCTION}

Forward scattering radar (FSR), is a sub-category of bistatic radars. When the target is moving near the baseline of the transmitter and the receiver, the bistatic angle is close to $180^{\circ}$ or in other words the target is moving in forward scattering (FS) region [1]. There are many advantages compared with monostatic radar, such as the radar cross section (RCS) enhancement which provides a better detection performance and also, stealth targets can be detected due to the independence of the RCS on the targets surface material. Shadow Inverse Synthetic Aperture Radar (SISAR) was first introduced by Chapurskiy in 1980s [2]. According to this theory, the FS signal of target in the Fresnel zone can be seen as the Fresnel transform of the target's complex profile function (CPF). From $\mathrm{CPF}$, the target's shadow profile can be extracted. A number of research projects are published based on the theory and applications of SISAR imaging including air target detection and automatic target classification [3] [4] [5]. However, more complex scenarios such as moving transmitter and receiver SISAR, are never considered. For example, in a previous work for CubedSAT based passive bistatic radar for space debris detection and characterization [6], potential SISAR imaging can be investigated. As a result, the need of a new model based on ME must be considered.

In FSR, to obtain the RHS, a signal synchronous processing is needed, which sometimes is not practical. The input signal at the receiver, will be the sum of the direct and the scattered signal coming from the transmitter and the target respectively and by means of an amplitude detector the one quadrature component of RHS can be extracted [1] . Hence, the need of RHS reconstruction can be considered as a major problem for FSR processing. In [7], the Hilbert transform (HT) is applied to obtain the analytical FS signal, whereas in [8], a segmented processing followed by HT and second order main lobe fitting was introduced. However these methods were designed for a simplified scenario in which transmitter and receiver where stationary. In this work it is shown that, in a case where a direct Doppler shift introduced by the relative motion of the transmitter and the receiver, the received FS signal can be modelled as a single-sided chirp. Therefore, the HT conditions are satisfied and thus, RHS reconstruction can be extracted effectively.

The remainder of the paper is organised as follows. Section II describes the classic SISAR imaging and the topology of FSR scenario. Section III introduces the moving-ends SISAR (ME-SISAR) model and the resolution ability, while section IV presents the novel approach of the RHS reconstruction. In Section $\mathrm{V}$ simulated results are reported to show the effectiveness and the conditions for this approach. Finally, Section VI concludes the paper.

\section{Classic SISAR}

In this section the fundamental principles of FSR and its topology are introduced in subsection A, followed by SISAR Imaging principles in subsection B.

\section{A. FSR Topology}

The considered FSR topology is shown in Fig. 1. The transmitter is placed at the origin of coordinates $(X, Y, Z)$, while the receiver is placed at the point $\mathrm{RX}(0, L, 0)$. The coordinates $\left(x^{\prime}, y^{\prime}, z^{\prime}\right)$ are parallel to coordinates $(X, Y, Z)$, and its origin $\left(x_{p}, y_{p}, z_{p}\right)$ represents the target's geometrical centre. The target moves in the plane parallel to the plane $X Y$ with velocity $V$, whose direction relative to the baseline is determined by the angle $\phi$. Angles $\alpha_{v}, \beta_{v}$ and $\alpha_{h}, \beta_{h}$ are the elevation and azimuth angles with respect to transmitter and receiver; $\alpha_{1}, \alpha_{2}$ are diffraction angles, while $d_{T}$ and $d_{R}$ are the distance from transmitter and receiver to the crossing point $C$ respectively. Due to the motion of the target, the bistatic distances $r_{c 1}, r_{c 2}$, will vary over the time introducing a Doppler shift on the scattered signal. From [9], the FS signal at the input of the receiver can be expressed in the form:

$$
S_{r}(t)=A(t) \sin (\psi(t))
$$




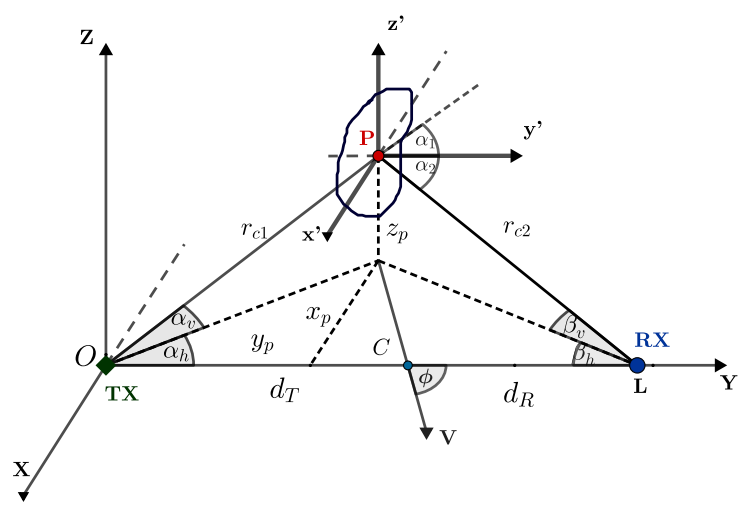

Fig. 1: FSR Topology

where the envelope $A(t)$ is defined by propagation losses and forward scatter cross section (FSCS), whereas the time varying phase $\psi(t)$ is dependent on the target's motion and is given by:

$$
\psi(t)=\frac{2 \pi}{\lambda}\left(r_{c 1}(t)+r_{c 2}(t)\right)
$$

where $\lambda$ is the wavelength of the transmitted signal.

\section{B. SISAR Imaging}

When the target's dimensions are much larger than the wavelength, by the Fresnel-Kirchhoff diffraction formula, the FS signal can be written as [10]:

$$
\dot{E}(t)=\frac{-j A}{2 \lambda} \iint_{S} \epsilon_{\tau}\left(x^{\prime}, z^{\prime}\right) \frac{e^{j k\left(r_{1}+r_{2}\right)}}{r_{1} r_{2}}\left(\cos \alpha_{1}+\cos \alpha_{2}\right) d S
$$

where $A$ is the transmitting signal amplitude, $k=2 \pi / \lambda, S$ is the shadow aperture and $r_{1}$ and $r_{2}$ are the distances from the transmitting source to every point of the target and from that point to the receiver respectively. The values of $r_{1}$ and $r_{2}$ can be evaluated as:

$$
\begin{gathered}
r_{1}=\sqrt{\left(x^{\prime}+x_{p}\right)^{2}+y_{p}^{2}+\left(z^{\prime}+z_{p}\right)^{2}} \\
r_{2}=\sqrt{\left(x^{\prime}+x_{p}\right)^{2}+\left(L-y_{p}\right)^{2}+\left(z^{\prime}+z_{p}\right)^{2}}
\end{gathered}
$$

Additionally, $\epsilon_{\tau}$ is the indicator function of the shadow profile of the target.

$$
\epsilon_{\tau}= \begin{cases}1, & \left(x^{\prime}, z^{\prime}\right) \in S \\ 0, & \left(x^{\prime}, z^{\prime}\right) \notin S\end{cases}
$$

The SISAR model obtained from [2], was based on the assumptions that diffraction angles are small, the object crosses the baseline perpendicularly and that the target is moving with constant velocity and thus the Doppler rate is constant without taking into consideration the high-order Doppler phases. Under these assumptions, the time domain FS signal is given as:

$$
\dot{E}(t)=\dot{Q} \int_{-\infty}^{\infty} \dot{H}\left(x^{\prime}\right) \exp \left[j \frac{\gamma}{2}\left(\frac{x^{\prime}}{V}+t\right)^{2} d x^{\prime}\right]
$$

where

$$
\begin{gathered}
\dot{Q}=\frac{A \sin \phi}{j \lambda r_{c 1} r_{c 2}} \exp \left(j 2 \pi \frac{L}{\lambda}+j \Omega^{2} z_{p}^{2}\right) \\
\gamma=2 \Omega^{2} V^{2} \sin ^{2} \phi \\
\Omega=\sqrt{\frac{\pi}{\lambda}\left(\frac{1}{d_{T}}+\frac{1}{d_{R}}\right)}
\end{gathered}
$$

and CPF can be modelled as:

$$
\dot{H}\left(x^{\prime}\right)=\int_{-\infty}^{\infty} \epsilon\left(x^{\prime}, z^{\prime}\right) \exp \left(j \Omega^{2} z^{\prime 2}+2 j \Omega^{2} z_{p} z^{\prime}\right) d z^{\prime}
$$

The CPF can be extracted by applying the inverse transform on (6) and compensate for $\gamma$ rate and target velocity $V$ component as:

$$
\dot{H}\left(x^{\prime}\right)=\frac{\gamma}{2 \pi v \dot{Q}} \int_{\eta} \dot{E}(t) \exp \left[-j \frac{\gamma}{2}\left(\frac{x^{\prime}}{v}+t\right)^{2}\right] d t
$$

where, $\eta$ is the observation time interval.

\section{MOVING-ENDS SISAR MODEL}

In this section we introduce a novel model for ME-SISAR. A more general approach is applied in order to extend the classic SISAR model for a more complex cases where the transmitter and/or the receiver are non-stationary objects. The topology in such scenario is shown in Fig. 2. All system components (target, transmitter and receiver) are randomly moving in space with the target moving in a plane which is parallel to the $X Y$ plane and with an angle $\phi$ relative to the baseline, the receiver is moving with an angle $\delta$ in respect to the baseline $L$ and the transmitter on the $X$-axis. At time zero all three components are aligned with the transmitter position at the origin of the $(\mathrm{X}, \mathrm{Y}, \mathrm{Z})$ and the receiver is located at $(0, L, 0)$. Assuming the transmitter's position as reference point, in a way that is moving on $X$-axis, the SISAR algorithm for ME is derived in this section.

The assumption of small diffraction angles is made for this model and thus the term from (3) can be approximated:

$$
\left(\cos \alpha_{1}+\cos \alpha_{2}\right) \approx 2
$$

The two distance variables from (4) and (5), can be approximated as:

$$
\begin{gathered}
r_{1} \approx r_{c 1}+\frac{x^{\prime 2}+2 x^{\prime}\left(x_{p}-X_{T}\right)+z^{\prime 2}+2 z^{\prime} z_{p}}{2 r_{c 1}} \\
r_{2} \approx r_{c 2}+\frac{x^{\prime 2}+2 x^{\prime}\left(x_{p}-X_{R}\right)+z^{\prime 2}+2 z^{\prime} z_{p}}{2 r_{c 2}}
\end{gathered}
$$




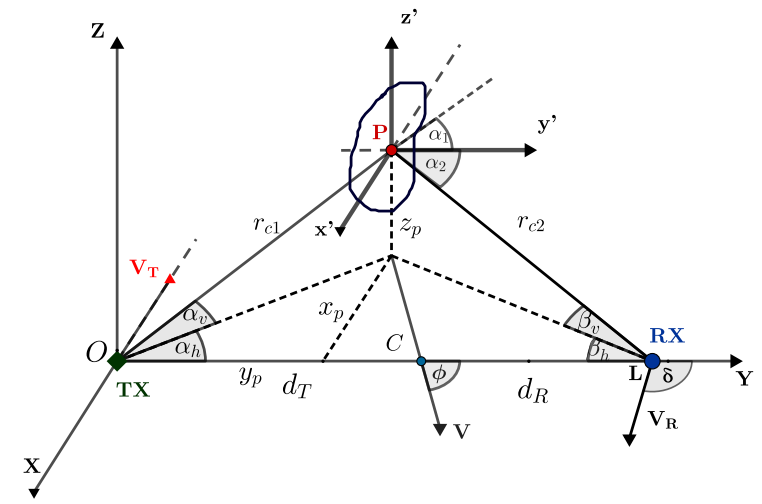

Fig. 2: Moving-ends Topology

where $r_{c 1}, r_{c 2}$ are the distances of the transmitter and receiver from the target's centre (see Fig.2) and their true values are given by:

$$
\begin{gathered}
r_{c 1}=\sqrt{\left(X_{T}-x_{p}\right)^{2}+y_{p}^{2}+z_{p}^{2}} \\
r_{c 2}=\sqrt{\left(X_{R}-x_{p}\right)^{2}+\left(Y_{R}-y_{p}\right)^{2}+z_{p}^{2}}
\end{gathered}
$$

where, $\left.(X-Y-Z)\right|_{T / R}$ are the transmitter/ receiver's time coordinates.

Referring to the diffraction formula in (3), the FS signal for the ME model can be derived as:

$$
\begin{array}{r}
\dot{E}=\frac{A e^{j k\left(r_{c 1}+r_{c 2}\right)}}{j \lambda r_{c 1} r_{c 2}} \int \dot{H}\left(x^{\prime}\right) e^{j k x^{\prime 2}\left(\frac{1}{2 r_{c 1}}+\frac{1}{2 r_{c 2}}\right)} \\
e^{j k x^{\prime} x_{p}\left(\frac{1-\frac{X_{T}}{x_{p}}}{r_{c 1}}+\frac{1-\frac{X_{R}}{x_{p}}}{r_{c 2}}\right)} d x^{\prime}
\end{array}
$$

where,

$$
\dot{H}\left(x^{\prime}\right)=\int \epsilon\left(x^{\prime}, z^{\prime}\right) e^{j k z^{\prime 2}\left(\frac{1}{2 r_{c 1}}+\frac{1}{2 r_{c 2}}\right)} e^{j k z^{\prime} z_{p}\left(\frac{1}{r_{c 1}}+\frac{1}{r_{c 2}}\right)} d z^{\prime}
$$

To obtain the time domain RHS, the motion equation for all components are applied: $x_{p}=V t \sin \phi, X_{T}=V_{t} t, X_{R}=$ $V_{R} t \sin \delta$

$$
\dot{E}(t)=\dot{Q} \int \dot{H}\left(x^{\prime}\right) e^{j \alpha x^{\prime 2}} e^{j \frac{\beta}{V} x^{\prime} t} d x^{\prime}
$$

with

$$
\begin{gathered}
\alpha=k\left(\frac{1}{2 r_{c 1}}+\frac{1}{2 r_{c 2}}\right), \quad \dot{Q}=\frac{A \sin \phi e^{j k\left(r_{c 1}+r_{c 2}\right)}}{j \lambda r_{c 1} r_{c 2}} \\
\beta=k V^{2} \sin \phi\left(\frac{1-\frac{V_{T}}{V \sin \phi}}{r_{c 1}}+\frac{1-\frac{V_{R} \sin \delta}{V \sin \phi}}{r_{c 2}}\right)
\end{gathered}
$$

The CPF of the target can be extracted using the inverse transform on (14), in the form of:

$$
\dot{H}\left(x^{\prime}\right)=\frac{\beta}{2 \pi V \dot{Q}} e^{-j \alpha x^{\prime 2}} \int_{\eta} \dot{E}(t) e^{-j \frac{\beta}{V} x^{\prime} t} d t
$$

\section{A. Resolution Ability}

The resolution for ME-SISAR can be evaluated using (15). The interval in this equation is a Fourier transform. Therefore, passing from frequency resolution $\delta \omega=\frac{2 \pi}{\eta}$ to $\delta x^{\prime}$ resolution, we have that:

$$
\omega=\frac{\beta}{V} x^{\prime} \quad \Longrightarrow \quad \delta x^{\prime}=\frac{2 \pi V}{\beta \eta}
$$

and by substituting $\beta$ from (14), the SISAR resolution can be evaluated as:

$$
\delta x^{\prime}=\frac{\lambda \sigma(1-\sigma) L}{\eta\left(V \sin \phi-\left[\sigma V_{R} \sin \delta+(1-\sigma) V_{T}\right]\right)}
$$

where $L$ is the baseline length and $\sigma$ is a scale factor, $0<$ $\sigma<1$, which expressing the crossing point $C$ in terms of the baseline length $L$ at the crossing time. Clearly, the resolution in $\mathrm{ME}$ scenario improves if the relative velocity component in $X$-axis direction between the target and the two ends, is increased.

\section{RHS RECONSTRUCTION}

As mentioned before, the reconstruction of RHS, is a very important procedure for SISAR imaging. To obtain the complex RHS signal from the carrier, synchronous processing is needed. Consequently, as it is widely used, exploiting the one quadrature component of the RHS, extracted by means of an amplitude detector, the analytical complex RHS signal can be reconstructed applying HT on one quadrature component.

The HT states that an analytical complex signal can be reconstructed from the signal's real part under the condition that the real amplitude and frequency modulated signal in the form:

$$
S(t)=I(t) \cos (\theta(t))
$$

has the spectrum of $\cos (\theta(t))$ outside the spectrum of its envelope $I(t) \in\left[-f_{0}, f_{0}\right][11]$.

In forward scattering, the Doppler signature of a target crossing the baseline has the form of double-sided chirp signal and at crossing time the Doppler is zero [12]. Consequently, a forward scattering Doppler signature cannot be used directly to reconstruct the analytical RHS signal.

\section{A. ME Impact on Doppler Signature}

In the case of ME scenario, it is possible that the Doppler of the target can stay away from zero for all the observation time interval. Using the geometry in Fig. 2 and (11), and (12), the Doppler shift of the target in time can be expressed as:

$$
\begin{aligned}
& f_{d}(t)=-\frac{1}{\lambda} \frac{d\left(r_{c 1}(t)+r_{c 2}(t)\right)}{d t} \\
& =-\frac{1}{\lambda}\left(\frac{\kappa t+c}{\sqrt{\kappa t^{2}+2 c t+d_{T}^{2}}}+\frac{\kappa_{1} t+c_{1}}{\sqrt{\kappa_{1} t^{2}+2 c_{1} t+d_{R}^{2}}}\right)
\end{aligned}
$$

where, 


$$
\begin{aligned}
\kappa & =V_{T}^{2}+V^{2}+2 V_{T} V \sin \phi \\
c & =d_{T} V \cos \phi \\
\kappa_{1} & =V_{R}^{2}+V^{2}-2 V_{R} V \cos (\phi-\delta) \\
c_{1} & =d_{R}\left(V_{R} \cos \delta-V \cos \phi\right)
\end{aligned}
$$

From (16), the instantaneous Doppler shift at crossing time $(t=0 s)$ is dependent on the receiver's velocity component on the $Y$-axis direction and is equal to:

$$
f_{d}(0)=\frac{-1}{\lambda}\left(\frac{c}{d_{T}}+\frac{c_{1}}{d_{R}}\right)=-\frac{V_{R}}{\lambda} \cos \delta
$$

Therefore, it is feasible to obtain a Doppler signature of a target, not in the double-sided chirp form but in a single-sided form instead, by introducing a receiver velocity component such as, the Doppler shift of the target is never crossing $0 \mathrm{~Hz}$ in the observation time. Consequently, the in-phase quadrature component obtained after envelope detection can satisfy the HT condition discussed before and therefore, the complex RHS signal can be reconstructed with minimum reconstruction error.

Fig. 3, illustrates the Doppler variation of a target in an observation time interval $\eta$, while the target is approaching, crossing and leaving the baseline always assuming that both ends are non-stationary objects. As shown in (18), the Doppler shift at the crossing time, is proportional to the receiver velocity component and thus is equal to zero if $V_{R}=0$ or $\delta \pm \frac{\pi}{2}$.

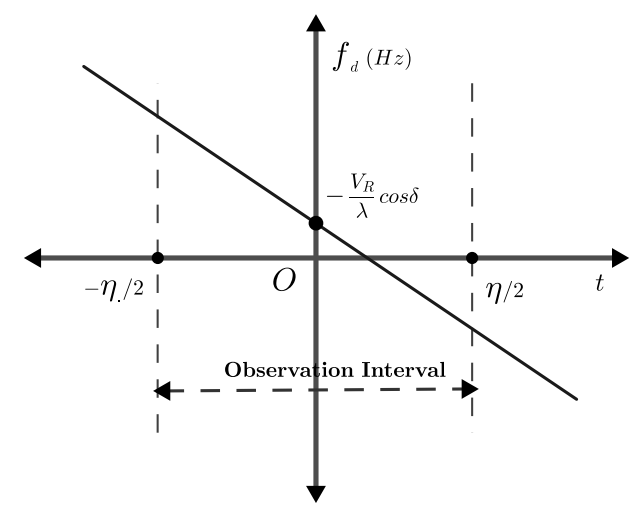

Fig. 3: Doppler Variation of Target Crossing the Baseline

From Fig. 3, in order to obtain a Doppler signature which satisfies the HT condition, the value of $\zeta=-\frac{V_{R}}{\lambda} \cos \delta$, must be chosen properly, in terms of system geometry and configuration, such as the Doppler shift never crosses $0 \mathrm{~Hz}$, and this is the case only when:

$$
\begin{aligned}
\text { if } \zeta>0 \text {, and } f_{d}(\eta / 2) & >0 . \\
\text { if } \zeta<0 \text {, and } f_{d}(-\eta / 2) & <0 .
\end{aligned}
$$

Therefore, depending on all the variables included in (16), the above condition in (19) must be satisfied in order to obtained the single-sided chirp form of the Doppler signature. Finally, the reconstructed RHS signal will be in the form of:

$$
\hat{\dot{E}}=\Re(\dot{E})+j(H T\{\Re(\dot{E})\})
$$

where, $\Re($.$) denotes the real part operator and H T($.$) , the HT.$

\section{Simulation Results}

In this section, the impact on the received Doppler signature based on the transmitter and receiver motion will be shown with simulated results. The target profile shown in Fig. 4 is used and Table I shows all the parameters assumed in this scenario. The real and imaginary part of the simulated

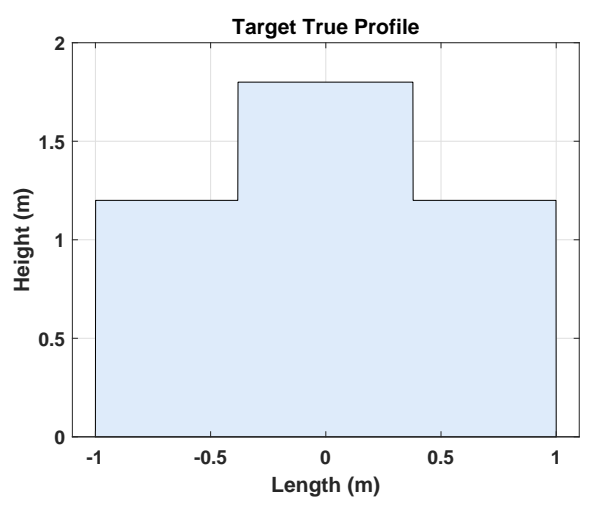

Fig. 4: Target Profile

TABLE I: Simulation Parameters

\begin{tabular}{|cc|}
\hline Wavelength & $0.193 \mathrm{~m}$ \\
\hline Baseline $(\mathbf{L})$ & $270 \mathrm{~m}$ \\
\hline Target Velocity & $30 \frac{\mathrm{m}}{\mathrm{s}}, \phi=80^{\circ}$ \\
\hline Receiver Velocity & $18 \frac{\mathrm{m}}{\mathrm{s}}, \delta=-10^{\circ}$ \\
\hline Transmitter Velocity & $5 \frac{\mathrm{m}}{\mathrm{s}}$ \\
\hline Observation Time & $1.5 \mathrm{~s}$ \\
\hline Crossing Point & $0.6 \mathrm{~L}$ \\
\hline Tx position at cros. time & $(0,0,0)$ \\
\hline Rx position at cros. time & $(0, L, 0)$ \\
\hline Target position at cros. time & $(0,0.6 \mathrm{~L}, 0)$ \\
\hline
\end{tabular}

RHS signal using (14), are shown in the Fig. 5 and Fig. 6 respectively.

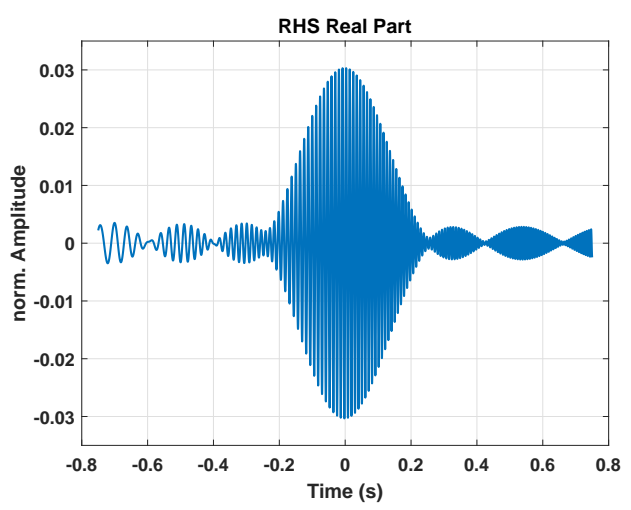

Fig. 5: Simulated RHS Real Part

As it is shown in the Fig. 5, and 6, the two quadrature components of RHS have the form of single-sided chirp signals. 


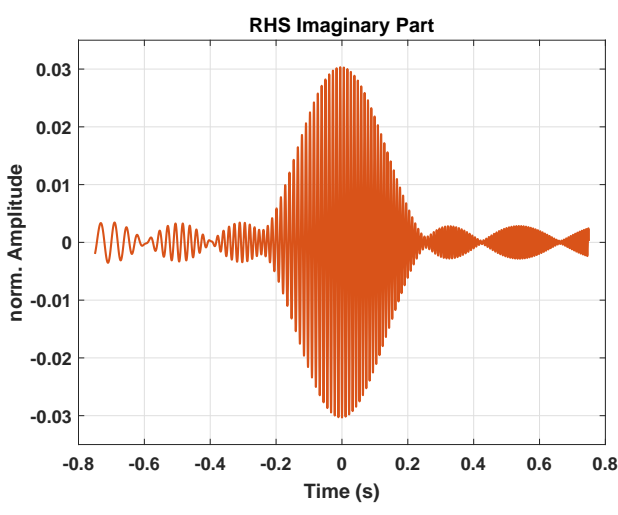

Fig. 6: Simulated RHS Imaginary Part

The direct Doppler introduced from the receiver shifted the Doppler frequency away from zero for all the observation time, and as a result the double-sided chirp form of the Doppler signature becomes a single-sided chirp. This can be seen from the spectrogram of the received signal shown in Fig. 7. Using

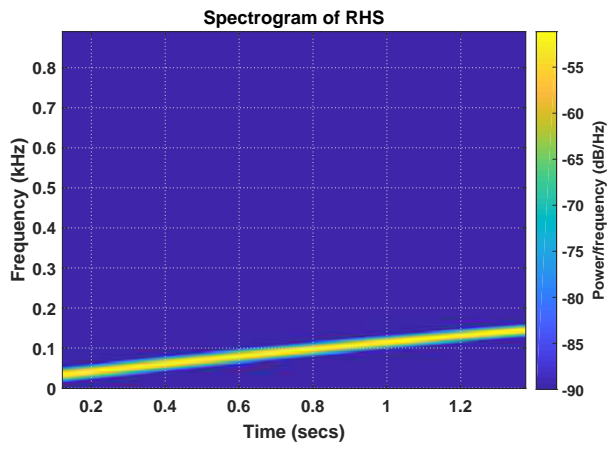

Fig. 7: Spectrogram of the received Signal

(16) it can be obtained that the Doppler shift varies within the interval $[-20,-160] \mathrm{Hz}$ during the observation time and therefore, there is not $0 \mathrm{~Hz}$ crossing. At the crossing time, the Doppler is proportional to the receiver velocity component as discussed in the Fig. 3. As a result, the analytical complex RHS signal can be reconstructed using HT directly without any segmented processing or main lobe fitting. The simulated and reconstructed imaginary parts are shown in the Fig. 8 and the reconstruction error in Fig. 9; to estimate the reconstructed imaginary part, (20) is used. The reconstructed RHS signal is almost ideal, with the reconstruction error going almost 0 in the middle area of the Fig. 9. The extracted image results are shown in the Fig. 10, using (15). Both simulated and reconstructed RHS are used to obtain the CPF of the target and plotted on the same figure.

\section{A. Matched Filtering}

After RHS reconstruction, to extract motion parameters of the target using a matched filtering technique, a bank of reference functions is needed [13], [9], [14]. Assuming both transmitter and receiver trajectories are known, a reference function for matched filtering will be in the form of:

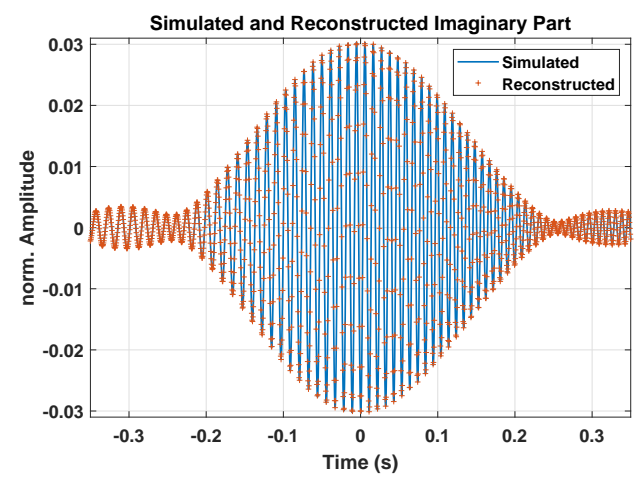

Fig. 8: Simulated and Reconstructed Imaginary Parts

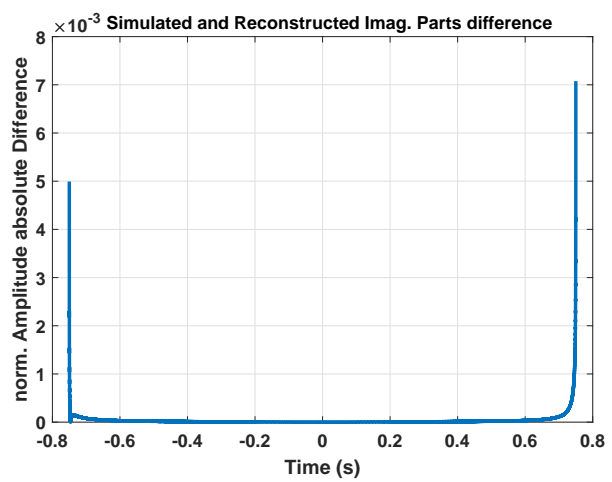

Fig. 9: Simulated and Reconstructed Imag. Parts difference

$$
S_{r e f}(t)=\hat{A}(t) \sin \left(\frac{2 \pi}{\lambda}\left[\hat{r}_{c 1}(t)+\hat{r}_{c 2}(t)\right]\right)
$$

Where $\hat{A}(t)$ is a window function as shown in [9], and $\hat{r}_{c 1}(t)$ and $\hat{r}_{c 2}(t)$, can be estimated using (11) and (12) for different target motion parameters.

In terms of matched filtering performance, is proper to compensate first for the direct Doppler that the receiver and transmitter introduced onto the received signal. The compensated RHS signal and the associated reference function can be estimated as:

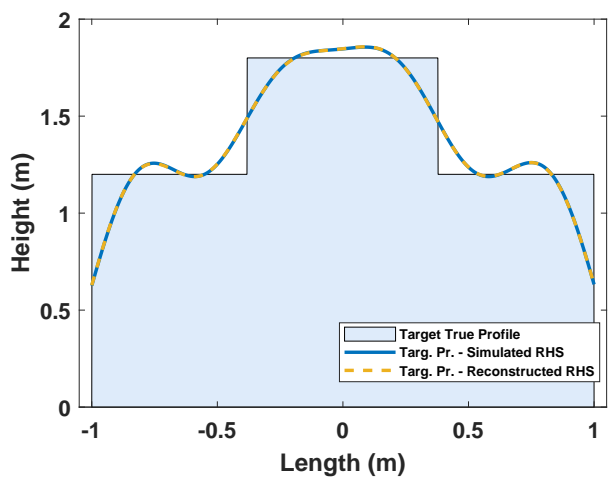

Fig. 10: Result Using Simulated and Reconstructed RHS 


$$
\begin{aligned}
\dot{E}_{\text {new }} & =\dot{E} e^{\frac{-j 2 \pi L(t)}{\lambda}} \\
S_{\text {refC }}(t) & =\hat{A}(t) \sin \left(\frac{2 \pi}{\lambda}\left[\hat{r}_{c 1}(t)+\hat{r}_{c 2}(t)-L(t)\right]\right)
\end{aligned}
$$

The spectrogram of the compensated RHS signal from Fig. 7 is illustrated in Fig. 11. The compensated RHS signal is a double sided chirp signal as expected.

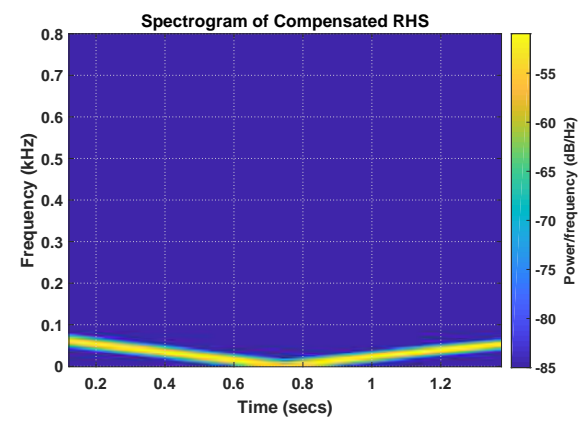

Fig. 11: Spectrogram of Compensated RHS

To evaluate, the effectiveness of the direct Doppler compensation on matched filtering performance, the matched filter technique is applied on the simulated RHS signal from Fig. 5; before and after direct Doppler compensation. Using a bank of reference functions evaluated from (21), (22), varying both target velocity and crossing angle, a map of the peak filter is illustrated in Fig. 12. The maxima in Fig. 12 are also normalized to the maximum autocorrelation output. The compensation of the direct Doppler on the RHS signal, clearly shows the effectiveness on the target's motion parameters extraction, by comparing the outputs of the two cases (see Fig. 12). The compensated RHS, effectively extracts the target's motion parameters (crossing angle and target's velocity, see Table I), whereas, the matched filter processing using the uncompensated RHS, is not efficient. From Fig. 12, it is shown that target's crossing angle $\left(\phi=80^{\circ}\right)$ and velocity $\left(V=30 \frac{m}{s}\right)$, are correctly estimated using the compensated RHS.

\section{Conclusion AND Future Work}

The presented work provides a novel approach for RHS reconstruction for SISAR imaging based on transmitter and receiver motion. Additionally, a model based on ME-SISAR is derived, which can also be applied in existing SISAR imaging applications. The simulated results showed that RHS signal can be almost ideally reconstructed exploiting the extra direct Doppler shift, the two ends introduce. The Doppler signature of the target transforms from a double sided to a single sided chirp signal, and therefore HT can be applied directly to the received FS signal. Future work consist of validation of the simulated results with experimental data.

\section{REFERENCES}

[1] M. E. Cherniakov, Bistatic Radar: Principles and Practice, 2007.
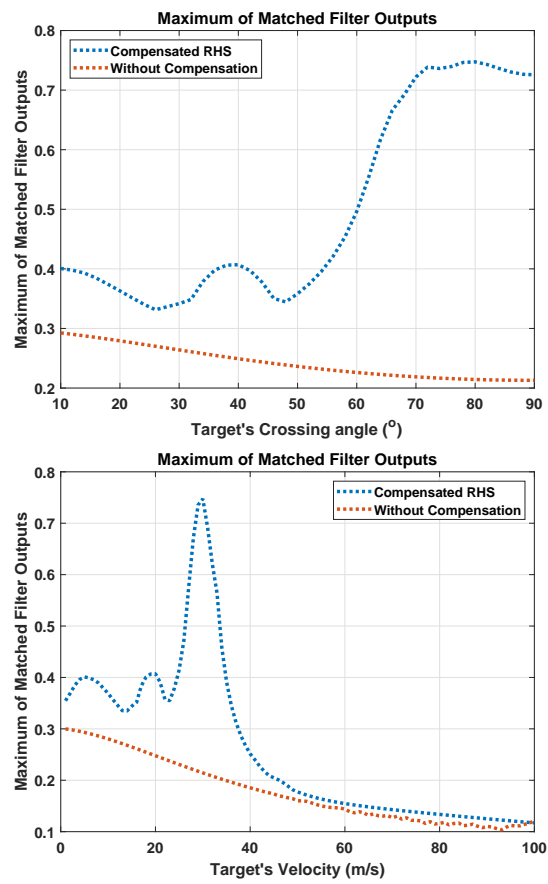

Fig. 12: Matched filter processing output

[2] V. V. Chapurskiy and V. N. Sablin, "Sisar: shadow inverse synthetic aperture radiolocation," in Record of the IEEE 2000 International Radar Conference [Cat. No. OOCH37037], May 2000, pp. 322-328.

[3] C. Hu, L. Wang, and C. Liu, "Sisar imaging method based on gnss signal: Theory and experimental results," in 2016 CIE International Conference on Radar (RADAR), Oct 2016, pp. 1-5.

[4] M. Cherniakov, R. S. A. R. Abdullah, P. Jancovic, M. Salous, and V. Chapursky, "Automatic ground target classification using forward scattering radar," IEE Proceedings - Radar, Sonar and Navigation, vol. 153, no. 5, pp. 427-437, Oct 2006.

[5] C. Yunhe, Z. Tao, L. Binfeng, and Z. Shouhong, "Experimental results for shadow inverse synthetic aperture radar," in 2009 IET International Radar Conference, April 2009, pp. 1-3.

[6] I. Theodorou, C. Clemente, and M. Vasile, "A passive debris tracking system in support to future space traffic management," in 15th Reinventing Space Conference, Glasgow UK, 2017.

[7] A. V. Myakinkov, "Optimal detection of high-velocity targets in forward scattering radar," in 2005 5th International Conference on Antenna Theory and Techniques, May 2005, pp. 345-347.

[8] C. Hu, C. Zhou, T. Zeng, and T. Long, "Radio holography signal reconstruction and shadow inverse synthetic aperture radar imaging in ground-based forward scatter radar: theory and experimental results," IET Radar, Sonar Navigation, vol. 8, no. 8, pp. 907-916, October 2014.

[9] M. Gashinova, L. Daniel, V. Sizov, E. Hoare, and M. Cherniakov, "Phenomenology of doppler forward scatter radar for surface targets observation," IET Radar, Sonar Navigation, vol. 7, no. 4, pp. 422-432, April 2013.

[10] E. W. Max B, Principles of Optics, 1999.

[11] F. J. T. Glenn Zelniker, Advanced Digital Signal Processing: Theory and Applications, 1993.

[12] C. Hu, V. Sizov, M. Antoniou, M. Gashinova, and M. Cherniakov, "Optimal signal processing in ground-based forward scatter micro radars," IEEE Transactions on Aerospace and Electronic Systems, vol. 48, no. 4, pp. 3006-3026, October 2012.

[13] C. Hu, M. Antoniou, M. Cherniakov, and V. Sizov, "Quasi-optimal signal processing in ground forward scattering radar," in 2008 IEEE Radar Conference, May 2008, pp. 1-6.

[14] A. D. Luca, L. Daniel, M. Gashinova, and M. Cherniakov, "Target parameter estimation in moving transmitter moving receiver forward scatter radar," in 2017 18th International Radar Symposium (IRS), June 2017, pp. 1-7. 